\title{
Research on Communication Security Technology in Intelligent \\ Substation
}

\author{
Wang lei, Ding Yan \\ Yellow River conservancy technical institute, Henan Kaifeng 475004 \\ wanglei@126.com
}

\begin{abstract}
Keywords: Intelligent substation; communication security technology; exchange technology; address binding
\end{abstract}

\begin{abstract}
With the rapid development of our country's economy, the per capita level of material life has improved significantly, the corresponding power consumption is also growing sharply, which brings a severe test to the stable operation of power grid system. As the mainstream trend of the current power grid construction, intelligent substation gathers many advanced technologies, wherein the communication technology plays a more and more significant role in it, and how to ensure the communication network security has become the currently primary work content. Thus, this article mainly analyzes the communication security technology in the intelligent substation, objectively sets forth the structure of the intelligent substation by integrating the actual situation, puts forward a safer and more reliable technical solution according to the characteristics of IED, so as to provide more solid guarantee for the intelligent substation, make sure the data transmission is securer, and promote the healthy and sustainable development of power utility.
\end{abstract}

\section{Introduction}

With the rapid development of science and technology, network communication technology has achieved a considerable success in improvement and innovation. Particularly in the current intelligent power grid construction, network communication technology and novel mutual induction technology are gradually becoming the core technologies. Due to its own characteristics, the intelligent substation gathers many advanced technologies and equipment, develops towards the all-station information digital and communication platform network directions, realizes the automatic acquisition, analysis, control, protection, etc. of data information, and realizes the intelligent control, online analysis and testing, collaborative interaction and other functions to the power grid according to the actual situation. Therefore, it is very necessary to strengthen the research on the communication security technology in the intelligent substation and provides a certain reference for the follow-up theoretical research and practice.

\section{Communication security problems existing in the substation}

(i) Network structure 
The substation mainly includes a station level, a bay level and a process level, wherein the station level mainly includes a back-stage system monitoring, fault information system and an engineer workstation; the bay level mainly includes a protector, a measuring and control device, an oscillograph, etc.; the process level includes an intelligent circuit breaker, electronic transformer, a merging unit, etc. The three levels of equipment are mutually connected via two levels of network, so as to realize the data transmission function among the three levels. Relevant fixed protection values, oscillograph documents, protection action and other information are transmitted via the station level network; the normal operation of the protection system, the on and off of the protection system, the position of the circuit breaker and other information[1] are transmitted via the process network.

\section{(ii) Message type}

First, production messages and specification messages oriented to the client server mode. Such types of messages mainly refer to messages communicating between the equipment on the station level and the bay level, the communication mode is an open 7-level system mode, so that the real-time requirement when the message information is transmitted is lower than that between the bay level and the process level. Second, substation event messages oriented to general objects. Such type of messages mainly transmit position signals, control signals, blocking signals, etc. between the bay level and the process level. Due to the transmission characteristics of the message information, the real-time requirement on the information is higher. Under normal circumstances, the messages are quite short, which brings smaller load to the network. When the system has faults, the message information is short and dense. Third, sampling messages. Such type of messages are mainly between the process level and the bay level in data information transmission, and realize the one-way transmission of the sampled data. Such form of messages has higher real-time requirement on the information. Different situations have different requirements on the length of the messages, but the network load is still kept stable[2].

\section{(iii) Communication security}

The data interaction of all devices within the substation is realized the communication of the merging unit. With the decrease of the quantity of measuring devices and protectors, the quantity of secondary cables is also decreased, and the overall design of the substation is simpler and simpler. However, for this solution still has a series of limited problems in security and reliability, the operation data of the protector is mainly attached on other communication networks and devices. Therefore, in case of any fault problems, the data cannot be transmitted, which will bring very serious consequences. For example, the data traffic flow of the sampling messages is quite high, the data is transmitted in the multicast form, so broadband recourses will be seriously wasted if no corresponding VLAN is classified. If the above-mentioned business is mixed with and networked with other business, the transmission of the network data will be blocked; or a piece of equipment has faults and sends abnormal message information, and such message information will have strong impact on other equipment.

\section{VLAN technology}

VLAN technology is also called as virtual local area network. It is mainly configuration management which is realized through the software function based on the traditional local area network technology, and can realize data interactive transmission in the virtual network. Different VLAN technologies may form different logical subnets, and can cover a lot of mobile terminal 
equipment. Such VLAN technology has very outstanding advantages, and can realize port isolation. For ports on the same section of switch, different VLANs can block the communication between the ports; the network is safer and more reliable, and different VLAN mobile equipment can realize direct communication, and the information transmitted in different broadcast domains will not be spread in the whole network; the management method is more flexible, and according to the subnet that a user belongs to, the confirmation and management[3] to the user network can be realized only by changing the port VLAN without changing physical connection.

In the intelligent substation, the sampling value network of the process level network is a multicast message. In order to prevent the waste of broadband resources, isolation can be realized by multicast swapping. Business functions can be classified between the station level and the bay level through the VLAN technology, they are classified in different voltage classes through the VLAN technology, and each bay is classified as one VLAN. The communication of 1 or more IEDs may occur in the intelligent substation network, which is realized through the VLAN technical communication function.

\section{Port address binding or locking}

The MAC address and port binding technology is in order to ensure the security and reliability of the port data transmission. After the MAC address and a port are bound, the network terminal equipment possessing the MAC address can only enjoy the network data transmission service via the bound port. If it is connected to other ports, it cannot access the Internet[4].

The port locking technology is similar to the binding technology: It is also about binding an MAC address with a special port, so as to improve the security of the port data transmission. After the port is locked, the network terminal equipment possessing the MAC address can only enjoy the network data transmission service via the bound port. If it is connected to other ports, it cannot access the Internet, and meanwhile the equipment cannot be connected with the Internet via the locked port. This locking technology is mainly based on the binding technology, and realizes the locking function by closing the locking port. In the intelligent substation, security protection can be provided for some very important business connection lines through the application port address binding or locking function, so as to prevent adverse impact brought by error operation, timely notify and report, and take reasonable countermeasures.

\section{Conclusion}

To sum up, in the construction of intelligent substation, as the core technology, how to ensure the security of the communication network is particularly critical, That needs the VLAN technology, port address binding or locking technology and multicast technology to provide security guarantee, so as to improve the security of communication network data transmission.

\section{References}

[1] Yang Liu. Communication Network System Design of Intelligent Substations [D]. North China Electric Power University (Baoding), 2011.

[2] Jinxiao Li, Yong Liao. Discussion on Application of Some Guarantee Technologies of Intelligent Substations [J]. M\&E Information, 2012,27(36):109-110.

[3] Wei Wu, Pengcheng He. Analysis to Communication Network Security of Intelligent Substations[J]. Theoretical Research on Urban Construction (Electronic), 2015,5(27):2845-2846. 
[4] Yonggang $\mathrm{Xu}$, Yang Feng, Chongchao Zhang, etc. Research on Communication Network Security Technology of Intelligent Substations[J]. Scientific \& Technological Innovation and Application, 2014,14(7):298-298,299. 